Research Paper

\title{
Resveratrol alleviate hypoxic pulmonary hypertension via anti-inflammation and anti-oxidant pathways in rats
}

\author{
Dunquan $\mathrm{Xu}^{1,2^{*}}$, Yan $\mathrm{Li}^{*}$, Bo Zhang ${ }^{*}$, Yanxia Wang1, Yi Liu1, Ying Luo ${ }^{1}$, Wen Niu1, Mingqing Dong1, \\ Manling Liu ${ }^{1}$, Haiying Dong ${ }^{1}$, Pengtao Zhao ${ }^{1}$, Zhichao Li ${ }^{1 凶}$ \\ 1. Department of Pathophysiology, Fourth Military Medical University, Xi`an, 710032, PR China. \\ 2. Clinical laboratory, the Eighth Hospital of PLA, Xigaze, 857000, PR China. \\ 3. Physical Examination Center of Beijing Military Region General Hospital, Beijing, 100700, PR China. \\ ${ }^{*}$ Contributed equally to this paper. \\ Corresponding author: Zhichao Li, MD, PhD. Department of Pathology and Pathophysiology, Fourth Military Medical University. E-mail: \\ pathophy@fmmu.edu.cn. Tel: 862984774548 Fax: 862984774548.
}

(1) Ivyspring International Publisher. Reproduction is permitted for personal, noncommercial use, provided that the article is in whole, unmodified, and properly cited. See http://ivyspring.com/terms for terms and conditions.

Received: 2016.07.11; Accepted: 2016.09.27; Published: 2016.11.23

\begin{abstract}
Resveratrol, a plant-derived polyphenolic compound and a phytoestrogen, was shown to possess multiple protective effects including anti-inflammatory response and anti-oxidative stress. Hypoxic pulmonary hypertension (HPH) is a progressive disease characterized by sustained vascular resistance and marked pulmonary vascular remodeling. The exact mechanisms of HPH are still unclear, but inflammatory response and oxidative stress was demonstrated to participate in the progression of $\mathrm{HPH}$. The present study was designed to investigate the effects of resveratrol on HPH development. Sprague-Dawley rats were challenged by hypoxia exposure for 28 days to mimic hypoxic pulmonary hypertension along with treating resveratrol $(40 \mathrm{mg} / \mathrm{kg} /$ day $)$. Hemodynamic and pulmonary pathomorphology data were then obtained, and the anti-proliferation effect of resveratrol was determined by in vitro assays. The anti-inflammation and anti-oxidative effects of resveratrol were investigated in vivo and in vitro. The present study showed that resveratrol treatment alleviated right ventricular systolic pressure and pulmonary arterial remodeling induced by hypoxia. In vitro experiments showed that resveratrol notably inhibited proliferation of pulmonary arterial smooth muscle cells in an ER-independent manner. Data showed that resveratrol administration inhibited HIF-1 $\alpha$ expression in vivo and in vitro, suppressed inflammatory cells infiltration around the pulmonary arteries, and decreased ROS production induced by hypoxia in PAMSCs. The inflammatory cytokines' mRNA levels of tumor necrosis factor $\alpha$, interleukin 6 , and interleukin $1 \beta$ were all suppressed by resveratrol treatment. The in vitro assays showed that resveratrol inhibited the expression of HIF-1 $\alpha$ via suppressing the MAPK/ERK 1 and PI3K/AKT pathways. The antioxidant axis of Nuclear factor erythroid-2 related factor 2/ Thioredoxin 1 (Nrf-2/Trx-1) was up-regulated both in lung tissues and in cultured PASMCs. In general, the current study demonstrated that resveratrol may prevent pulmonary hypertension through its anti-proliferation, anti-inflammation and antioxidant effects. Hence, the present data may offer novel targets and promising pharmacological perspective for treating hypoxic pulmonary hypertension.
\end{abstract}

Key words: resveratrol; hypoxic pulmonary hypertension; reactive oxygen species; inflammation; hypoxia inducible factor 1 a; estrogen receptor; nuclear factor erythroid-2 related factor 2; thioredoxin 1.

\section{Introduction}

Resveratrol polyphenolic compound, is one kind of phytoestrogen which plays important cardio-protective roles such as anti-oxidant, anti-inflammation, endothelial protection, induction of nitric oxide (NO) generation, and inhibition of platelet aggregation[1-5]. Recent years, studies found that resveratrol exerts inhibiting effects on vascular smooth muscle cells proliferation[6, 7]. Lots of studies showed that resveratrol also exerts liver protective effects such as anti-fibrosis, preventing hepatic steatosis, and ameliorating hepatotoxicity due to various etiological 
factors[8-10]. Moreover, the anti-tumor effects of resveratrol were investigated intensively these years[11-13].

Hypoxic pulmonary hypertension (HPH) is a subset of 5 types of pulmonary hypertension classified according to the guidelines for the diagnosis and treatment of pulmonary hypertension, which is characterized by persistent elevation of pulmonary arterial pressure and vascular remodeling[14]. Hypoxia initially induces acute pulmonary vasoconstriction, and gradually, vascular remodeling, which participate in the development of HPH[15]. Pulmonary vascular remodeling is a hallmark of the persistent exacerbation, and on this occasion, it's difficult to halt the progression of HPH. Vascular remodeling attributes to various cellular and molecular changes, which PASMCs proliferation is the key factor[15]. Moreover, studies showed that inflammation and oxidative stress play important roles in the development of $\mathrm{HPH}[16,17]$.

Current therapies on $\mathrm{HPH}$ mainly focus on vasoconstriction factors and only offer symptomatic relief (such as oxygen supporting, NO inhalation, calcium channel blockers, phosphodiesterase inhibitors, endothelin receptor antagonists, prostanoids, etc.), and few therapies could reverse the progression of HPH. Novel approaches treating HPH are urgent needed to develop and aim to halt or reverse the progression of pulmonary hypertension. Our previous studies showed that estrogen exerts anti-proliferating effects on PASMCs and effectively halts progression of vascular remodeling in rats[18].

Considering the potential pro-thrombus and myocardial infarction adverse effects of estrogen application[19, 20], we turn our eyes on resveratrol, one kind of phytoestrogen, which was demonstrated as safe application on animal models[21]. In view of the cardioprotective effects of resveratrol, we hypothesize that resveratrol exert anti-proliferation, anti-oxidant, and anti-inflammation effects during the progression of $\mathrm{HPH}$.

To verify our hypothesis, we explored long-term gavage administration of resveratrol in chronic hypoxia exposed rats. Chronic hypoxia resulted in significant elevation of right ventricular systolic pressure, vascular remodeling, PASMCs proliferation, infiltration of inflammatory cells in lungs, and increase of reactive oxygen species in rats. In the present study we addressed those questions whether resveratrol exert effects of inhibiting PASMCs proliferation, anti-inflammation, decreasing ROS production in rat models of chronic hypoxic pulmonary hypertension. Additionally, we further explored the mechanisms underlying the protective effects of resveratrol.

\section{Methods}

\section{Experimental groups and in vivo hypoxia}

Animals were randomly designated into 4 groups: 1) normoxia group, $n=6,2)$ normoxia group treated with resveratrol $(40 \mathrm{mg} / \mathrm{kg} /$ day, gavage administration), $n=6,3$ ) chronic hypoxia group, $n=6$, and 4) chronic hypoxia group treated with resveratrol (40 $\mathrm{mg} / \mathrm{kg} /$ day, gavage administration) $\mathrm{n}=6$. The appropriate dose of resveratrol was chose according to our previous studies (please see in the supplemental data). The normoxic groups were housed at ambient barometric pressure for 28 days (about $718 \mathrm{mmHg}, \mathrm{PO} 2$ is about $150.6 \mathrm{mmHg}$ ). The hypoxic groups were housed in a hypobaric hypoxia chamber depressurized to $380 \mathrm{mmHg}\left(\mathrm{PO}_{2}\right.$ was reduced to about $79.6 \mathrm{mmHg}$ accordingly) for 28 days. All animals were raised in a 12:12-hour's light-dark cycle condition, and were supplied with free food and water. The room temperature was maintained at 25 ${ }^{\circ} \mathrm{C}$, and the padding stuff was changed once a week.

\section{Hemodynamic analysis and tissue preparation}

After 28 days hypoxia exposure, the animals were fasted overnight and anesthetized with $20 \%$ ethylurethanm ( $4 \mathrm{~mL} / \mathrm{kg}$ i.p.). Next, the right jugular vein of rat was carefully isolated and a special-shaped catheter linked to the Powerlab system (AD Instruments, Bella Vista, NSW, Australia) was inserted into the right ventricle chamber through the right jugular vein, and the right ventricle systolic pressure (RVSP) was then recorded. Meanwhile, the mean carotid artery pressure (mCAP) was also recorded via a PE50 catheter inserted into the carotid artery. After the hemodynamic data were recorded, sternotomy surgery was performed, and the rats were perfused by paraformaldehyde. Then lungs together with heart were harvested. The right ventricle (RV) and left ventricle plus septum $(\mathrm{LV}+\mathrm{S})$ were weighed, and the ratio of $(\mathrm{RV} / \mathrm{LV}+\mathrm{S})$ was obtained as the right ventricle hypertrophy index (RVHI). Next, the rats' right ventricle walls were frozen-sectioned into $20 \mu \mathrm{m}$ for next analysis. The lungs from different animals were sectioned into 4-mm-thick slices at the same part (the lower lobe of the right lung) and soaked in 10\% formalin solution $(\mathrm{pH}=7.4)$. The remained parts were kept in a $-80^{\circ} \mathrm{C}$ freezer for next experiments.

\section{Hypoxia-induced right ventricle remodeling analysis}

Right ventricle remodeling was analyzed using immunofluorescence microscopy after incubation with wheat germ agglutinin conjugated to Oregon Green-488 (5 mg/mL in PBS, $10 \mathrm{~min}$; Invitrogen, Carlsbad, CA) and DAPI (1 mM in PBS; Beyotime Inc, 
Jiangsu, China). Myocytes were identified by their typical shape which stained by the wheat germ agglutinin of the cell membrane (glycocalyx is abundant in myocytes, and wheat germ agglutinin is a universal marker of glycocalyx). The cross sectional area of 100 myoctes in each group was then manually obtained in 10 consecutive visual fields by a pathologist, and myocytes hypertrophy was then estimated. The morphological analyses were conducted in a double-blind method.

\section{Hypoxia-induced pulmonary arterial remodeling analysis}

After fixation in formalin for 72 hours, the lung slices were embedded in paraffin and sectioned into 4 - $\mu$-thick sections. Immunohistochemistry for a-smooth muscle actin (1:800, Millipore, Bedford, MA, USA) was done to detect the vascular changes in pulmonary arterioles. Morphological analyses were also processed in a double blind method by a pathologist. Pulmonary arteries with external diameter ranged from 50 to $200 \mu \mathrm{m}$, total 40 arteries (5-10 per rat) were obtained from each group. The external and internal diameters, the cross sectional area of medial wall, and the total cross sectional vessel area of pulmonary arterioles were processed by Image-Pro Plus (Version 5.1, Media Cybernetics, Bethesda, MD, USA). Pulmonary vascular structure remodeling was assessed by $\mathrm{WA} \%=$ (wall area) $/($ vessel cross sectional area) $\times 100 \%$.

\section{Immunohistochemistry}

The prepared lung sections were incubated overnight at $4^{\circ} \mathrm{C}$ with anti-ED1 (1:200, Millipore, Bedford, USA) and phospho-NF-kB (1:50, Cell Signaling Technology, Inc., MA, USA) antibody, respectively. Slides were washed and incubated with corresponding secondary antibodies conjugated with alkaline phosphatase.

\section{Real-time PCR}

RNA was extracted from rat lung tissues by using the RNA Isolation Kit (Beyotime Inc, Jiangsu, China). Real-time PCR was performed to assess mRNA expression of the following genes. The primers for genes were as below, IL-1 $\beta$ : forward: 5'-CAACCAACAAGTGATATTCTCCATG-3',

reverse: 5'-GATCCACACTCTCCAGCTGCA-3'; IL-6: forward: 5'-TTCACAAGTCCGGAGAGGAG-3', reverse: $\quad 5^{\prime}$-ACTCCAGAAGACCAGAGCAG-3'; TNF-a: forward: 5'-CAAACCACCAAGCAGAGGAG -3', reverse: 5'-ATGAAGTGGCAAATCGGCTG-3'; VEGF: forward: 5'-GCTTTACTGCTGTACCTCCAC3', reverse: 5'-AGAAGTTCGGCAGGACAC-3'; and VEGF: forward: 5'-GCT TTA CTG CTG TAC CTC
CAC-3', reverse: 5'-AGA AGT TCG GCA GGA CAC-3'.

\section{Oxidative stress in vivo assays}

The $\mathrm{H}_{2} \mathrm{O}_{2}$ level represents the amount of oxidative stress in vivo. Commercial kits were employed to detect $\mathrm{H}_{2} \mathrm{O}_{2}$ level in rat lungs (Beyotime Inc, Jiangsu, China). According to the instructions, the rat lung tissues were homogenized in lysate and clarified by centrifugation $(12,000 \mathrm{rpm}, 10 \mathrm{~min}$ at 4 $\left.{ }^{\circ} \mathrm{C}\right)$. The supernatant was then collected and the level of $\mathrm{H}_{2} \mathrm{O}_{2}$ was detected using a spectrophotometer at $560 \mathrm{~nm}$ wavelength.

\section{Superoxide dismutase (SOD) and glutathione (GSH) activities in rat lungs}

The activities of SOD and GSH in rat lungs were assayed by using the commercial kits (Nanjing Jiancheng Bioengineering Institute, Jiangsu, China).

\section{Pulmonary arterial smooth muscle cells culture and hypoxia-induced cell proliferation assays}

Rat PASMCs were obtained by explants method as described previously[22]. Smooth muscle cell identity was verified by smooth muscle a-actin staining at each passage ( $>95 \%$ of cells stained positive for smooth muscle a-actin) and cells of passages 3-6 were used. PASMCs were divided into normoxia, hypoxia, and hypoxia plus resveratrol (concentrations of 10, 20, and $40 \mu \mathrm{mol} / \mathrm{L}$ of resveratrol were adopted), total 5 groups. The normoxic groups was maintained at $37^{\circ} \mathrm{C}$ in $95 \%$ O2, $5 \% \mathrm{CO}_{2}$ condition (HH CP-01W, Shanghai Boxun Industry \& Commerce Co., Ltd, Shanghai, China), and the hypoxic groups were cultured in 3\% oxygen condition for 48 hours (HERAcell 240, Heraeus Inc, Germany), separately. For further investigating whether the estrogen receptors involved in the anti-proliferation effect of resveratrol, estrogen receptors inhibitor ICI182780 was administered in the PASMCs. PASMCs were grouped into normoxia, hypoxia, hypoxia+resveratrol (40 $\mu \mathrm{mol} / \mathrm{L})$, hypoxia+ICI182780, and hypoxia+resveratrol\&ICI182780, total 5 groups. Optimal concentration of ICI182780 (100 nM) and ERs expression in PASMCs was tested in preliminary experiments. A commercial WST-1 cell proliferation and cytotoxicity assay kit (Beyotime Inc, Jiangsu, China) were used in the present study. The optical density values were detected at $450 \mathrm{~nm}$ with $690 \mathrm{~nm}$ reference wavelength by using a spectrophotometer (PowerWave XS, BioTek Inc, Vermont, USA).

Reactive oxygen species detection in PASMCs

A commercial DCF-DA fluorescence probe based 
ROS assay kit was adopted to analyze the production of ROS (Beyotime Inc, Jiangsu, China). In brief, cells were seeded in special dishes for next laser confocal microscopy detection. The cells were treated with or without different concentrations of resveratrol under hypoxia condition for 72 hours. Then the cells were washed and incubated with $10 \mu \mathrm{M}$ DCF-DA for 30 min at $37^{\circ} \mathrm{C}$. The intracellular ROS levels were determined by the relative fluorescence intensity using laser confocal microscopy. The $\mathrm{H}_{2} \mathrm{O}_{2}$ concentrations in PASMCs culture supernatant were also analyzed by using the commercial kits (Beyotime Inc, Jiangsu, China) mentioned above.

\section{Western blotting analysis}

Total lysates were obtained from harvested lung tissue and cultured PASMCs. Lung homogenates were prepared in RIPA lysis buffer (Beyotime Inc, Jiangsu, China). The protease inhibitor of phenylmethylsulfonyl fluoride (PMSF, $1 \mathrm{mM}$ ) was added to the RIPA buffer in advance. Equivalent amounts of protein $(30 \mu \mathrm{g})$ from each sample were separated on $12 \%$ SDS-polyacrylamide gels, and then transferred onto $0.22 \mu \mathrm{M}$ nitrocellulose filter membranes (Millipore, Bedford, USA). The primary antibodies were HIF-1 a (1:500; Millipore, Bedford, USA), anti-Nrf2 monoclonal antibody (1:200, Bioworld Technology, Nanjing, China), anti-Trx1 monoclonal antibody (1:1000, Epitomics biotechnology, California, USA), anti-phospho-AKT (1:500) and anti-AKT (1:1000) antibodies (Cell Signaling Technology, Inc, Massachusetts, USA), and anti-phospho-ERK (1:500) and anti-ERK antibodies (1:500). The signals were detected by WesternBright ECL kit (Advansta, CA, USA).

\section{Statistical analyses}

All values were expressed as mean $\pm \mathrm{SD}$. The statistical significance of differences between groups was evaluated by oneway analysis of variance (ANOVA), followed by LSD test for post hoc multiple comparisons (SPSS for Windows version 16.0, Chicago, USA). Significant difference was accepted at $P<0.05$.

\section{Results}

\section{Resveratrol treatment attenuated chronic hypoxia-induced right ventricle remodeling and right ventricular systolic pressure in rats}

After 28 days hypoxia exposure, the myocytes of right ventricle in hypoxia group was significantly enlarged versus the normoxic groups (Figure $1 \mathrm{a}$, $\mathrm{b}$; $P<0.01)$. Resveratrol administration notably inhibited the hypertrophy of myocytes of right ventricle after chronic hypoxia (Figure $1 \mathrm{a}, \mathrm{b} ; P<0.01$ ). There was no significant difference found in the two normoxic groups (Figure 1a, b). Accordingly, hypoxia exposure also markedly increased RVHI versus the normoxic groups (Figure 1c; $P<0.01$ ), and resveratrol treatment significantly decreased the elevation of RVHI (Figure $1 c, P<0.01)$. There was no significant difference found in the two normoxic groups (Figure 1c).

Chronic hypoxic condition also dramatically increased RVSP versus the normoxic groups (Figure $1 \mathrm{~d}, \quad P<0.01)$, and resveratrol treatment notably decreased the increased RVSP (Figure 1d, $P<0.01$ ). Similarly, there was no significant difference found in the two normoxic groups (Figure 1d).

\section{Resveratrol treatment attenuated chronic hypoxia-induced pulmonary arterials remodeling in rats}

Chronic hypoxia exposure resulted in thickened pulmonary arterial tunica media and accumulated extracellular matrix (Figure $2 \mathrm{aC}$ ). Compared with the hypoxic exposure group, resveratrol treatment obviously alleviated the deterioration of the pulmonary arterials remodeling (Figure 2aD). The changes of arterials remodeling were not found in the normoxic groups (Figure 2aA, B). Statistically, the pulmonary arterial structure remodeling indices (WA\%) and (WT\%) were both significantly elevated after chronic hypoxia exposure $(P<0.01$, Figure $2 b, c)$. Resveratrol administration significanlty decreased both indices of pulmonary arterial structure remodeling $(P<0.01$, Figure $2 b, c)$. Similarly, there were no significant differences between the normoxia and normoxia+res groups in both indices (Figure 2b, c).

\section{Resveratrol treatment reduced hypoxia exposure induced infiltration of neutrophils in rat lungs and the expression of phosphorylated nuclear factor $\mathbf{K B}$}

Chronic hypoxia resulted in plentiful infiltration of neutrophils in rat lungs compared to the normoxic groups $(P<0.01$, Figure $3 \mathrm{aC}, \mathrm{b})$. However, resveratrol treatment significantly reduced the infiltration of neutrophils in rat lungs $(P<0.01$, Figure $3 a D, b)$. There were few neutrophils found in both normoxic groups (Figure 3aA, B). Moreover, chronic hypoxia also resulted in significant expression of phosphorylated nuclear factor $\mathrm{kB}(\mathrm{NF}-\mathrm{kB})$ versus the normoxic groups $(P<0.01$, Figure $3 \mathrm{aE}, \mathrm{d})$, and resveratrol treatment notably inhibited its expression $(P<0.01$, Figure $3 \mathrm{aE}$, d). No positive expression of phosphorylated NF-kB was found in both normoxic groups (data not shown). 
a

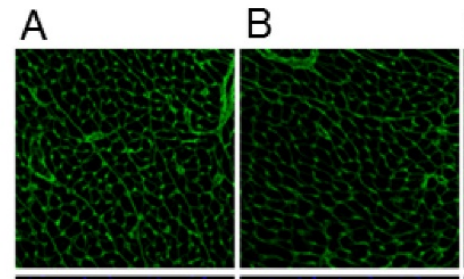

C

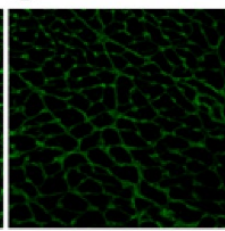

$\mathrm{D}$
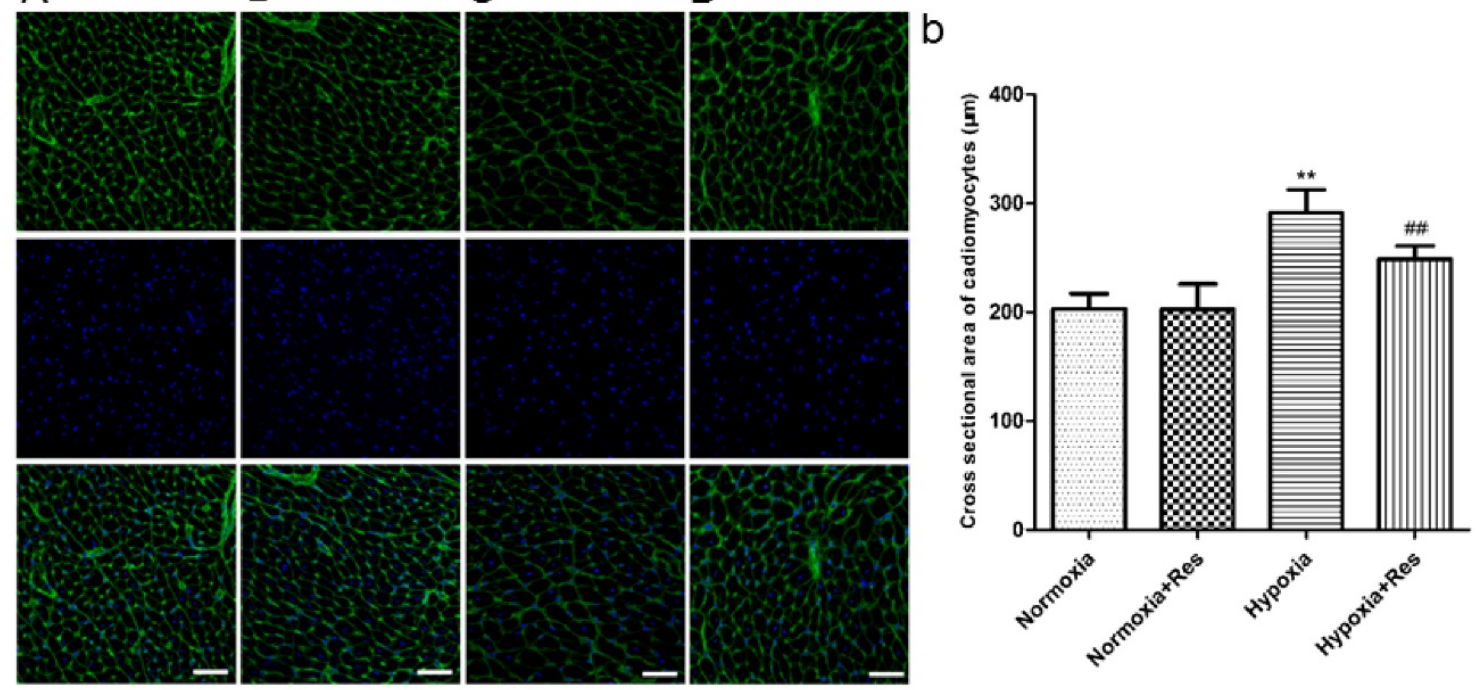

C

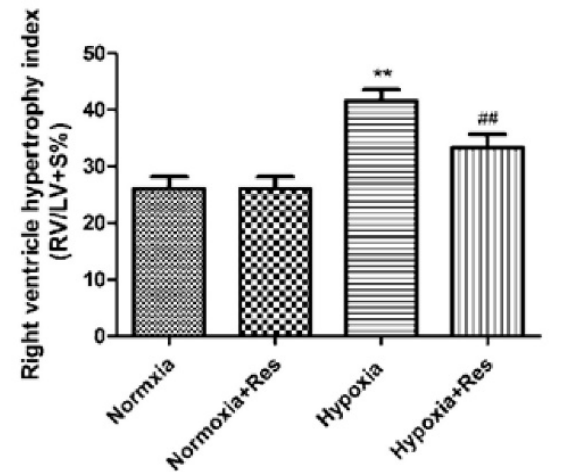

d

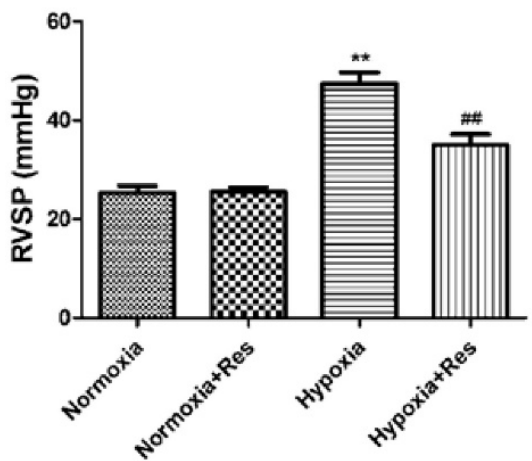

Figure 1. Staining of wheat germ agglutinin conjugated to Oregon Green-488 in rat cardiomyocytes, right ventricle hypertrophy index, and right ventricle systolic pressure.
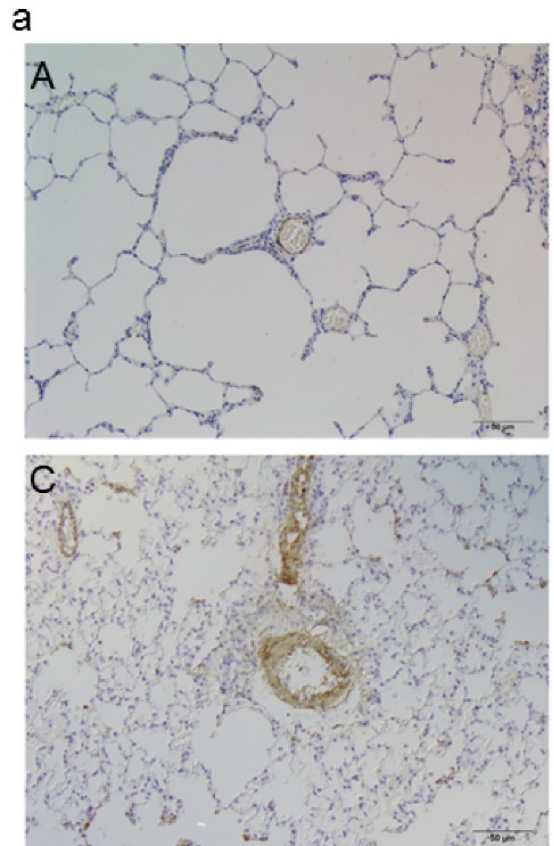
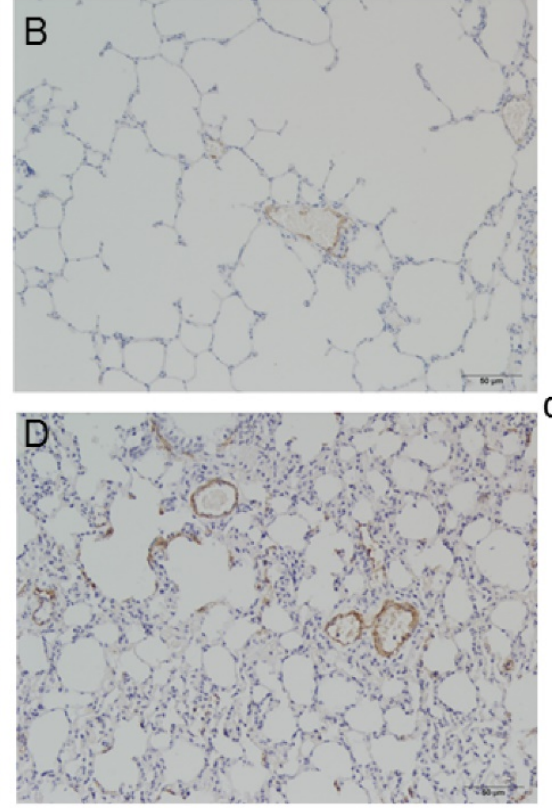

$b_{\text {วิำ }}$
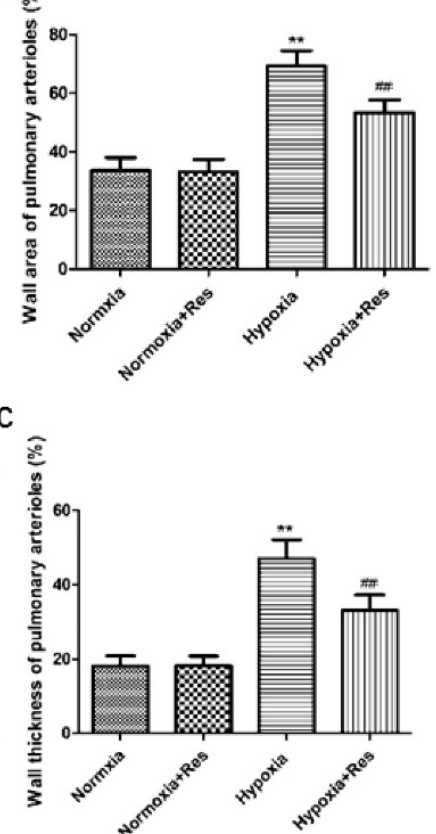

Figure 2. Immunohistochemistry staining of $\alpha$-smooth muscle actin in pulmonary arterioles. 
a

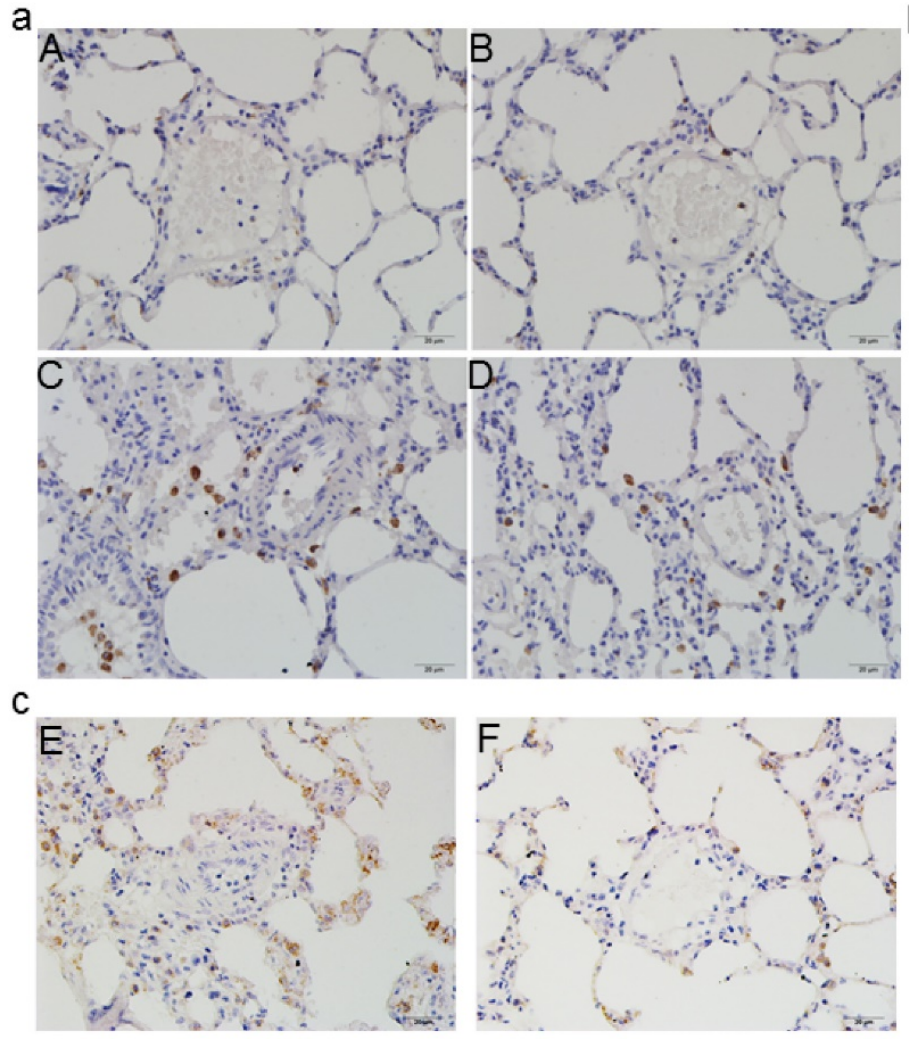

b

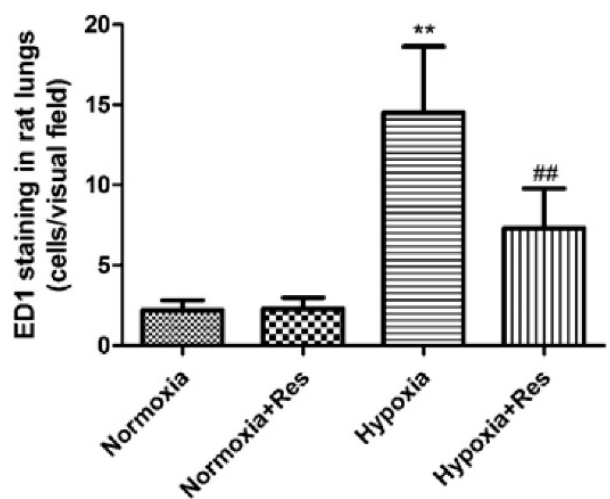

d

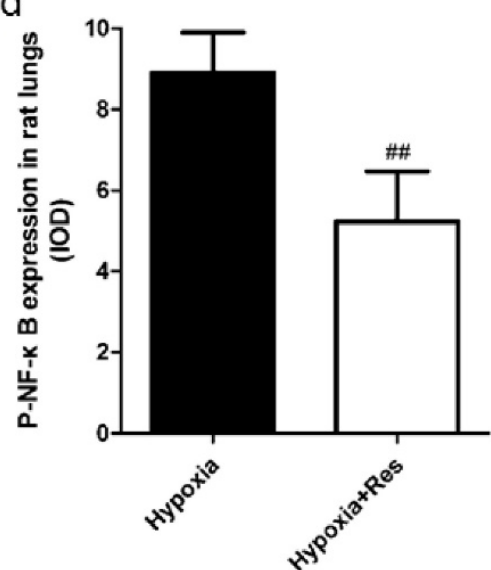

Figure 3. EDI and Phosphorylated nuclear factor KB immunohistochemistry staining in rat lungs.

\section{Resveratrol treatment decreased elevated expression of IL-6, IL-1 $\beta$, TNF- $\alpha$, and VEGF after hypoxia exposure}

Inflammatory factors IL-6, IL-1 $\beta$, TNF- $\alpha$ and cytokine VEGF were all significantly increased in mRNA levels after chronic hypoxia $(P<0.01$, Figure 4$)$, and all those factors were greatly decreased after resveratrol treatment $(P<0.01$, Figure 4$)$.

\section{Resveratrol treatment reduced hypoxia exposure induced production of $\mathrm{H}_{2} \mathrm{O}_{2}$ in rat lungs}

Compared to the normoxic groups, there was significant elevation of $\mathrm{H}_{2} \mathrm{O}_{2}$ found in rat lungs after 28 days hypoxic exposure $(P<0.01$, Figure 5), and resveratrol treatment significantly reduced its elevation in a dose-dependent way $(P<0.01$, Figure 5). No significant differences were found between the two normoxic groups (Figure 5).

\section{Resveratrol treatment reversed GSH and SOD reduction induced by hypoxia exposure}

Hypoxia exposure significantly decreased the GSH and SOD activities $(P<0.01$, VS normoxic groups; Figure 6), and resveratrol treatment reversed both of
GSH and SOD activity nearly to the normal levels $(P<0.01$, VS hypoxic group; Figure 6$)$.

\section{Resveratrol treatment inhibited hypoxia-induced proliferation of PASMCs in an ER-independent manner}

Significant proliferation of PASMCs was induced by hypoxia exposure compared to the normoxic group $(P<0.01$, VS normoxic group; Figure $7)$, and resveratrol notably inhibited the proliferated PASMCs in a dose-dependent way $(P<0.01$, Figure 7$)$. Moreover, resveratrol significantly inhibited the proliferation of PASMCs induced by hypoxia, and co-treated with the ER-inhibitor ICI182780 did not alter the anti-proliferation effect of resveratrol. Additionally, ICI182780 had no notable effect on the PASMCs under hypoxia exposure.

\section{Resveratrol decreased hypoxia-induced ROS and $\mathrm{H}_{2} \mathrm{O}_{2}$ production in vitro}

Hypoxia resulted in great increase production of ROS in the cultured PASMCs compared to the normoxic group $(P<0.01$, VS normoxic group; Figure $8 \mathrm{a}, \mathrm{b})$. However, resveratrol administration significantly inhibited the production of ROS in a 
dose-dependent way $(P<0.01$, VS hypoxic group; Figure $8 \mathrm{a}, \mathrm{b})$. Similarly, hypoxia also induced significant increased production of $\mathrm{H}_{2} \mathrm{O}_{2}$ in cell cultured supernatant $(P<0.01$, VS normoxic group;
Figure 8c), and resveratrol administration significantly inhibited the production of $\mathrm{H}_{2} \mathrm{O}_{2}$ in a dose-dependent way $(P<0.01$, VS hypoxic group; Figure 8c).
A
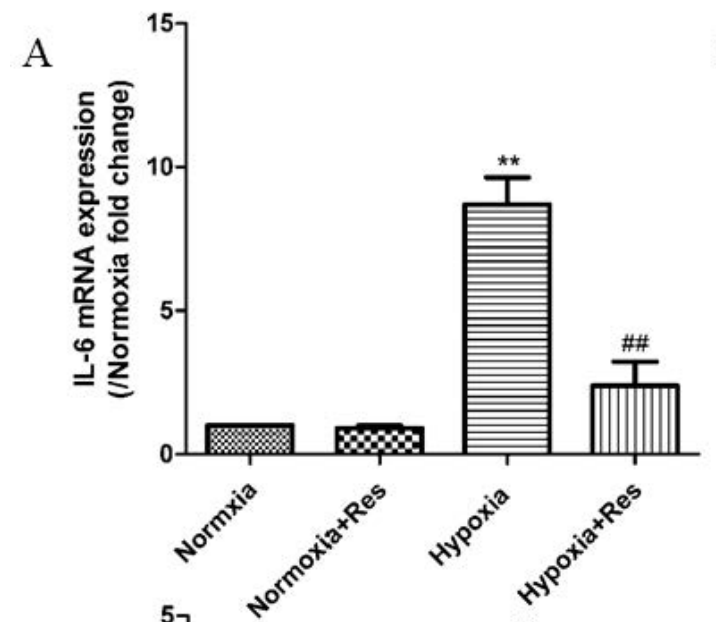

C

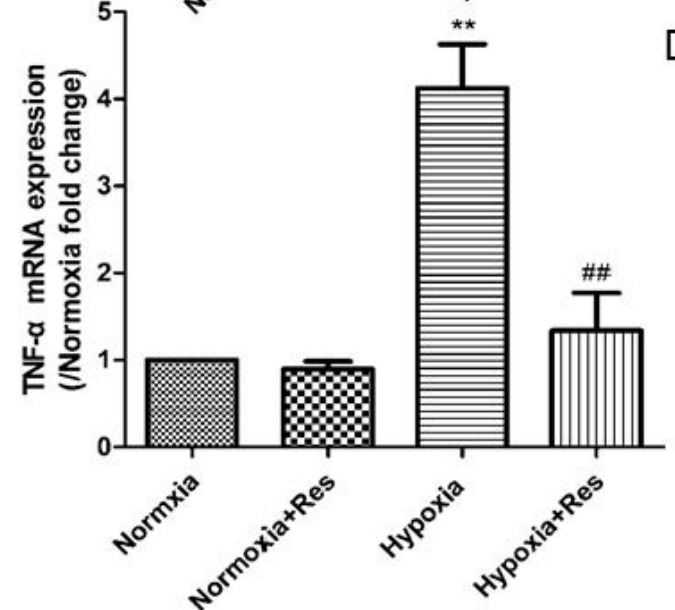

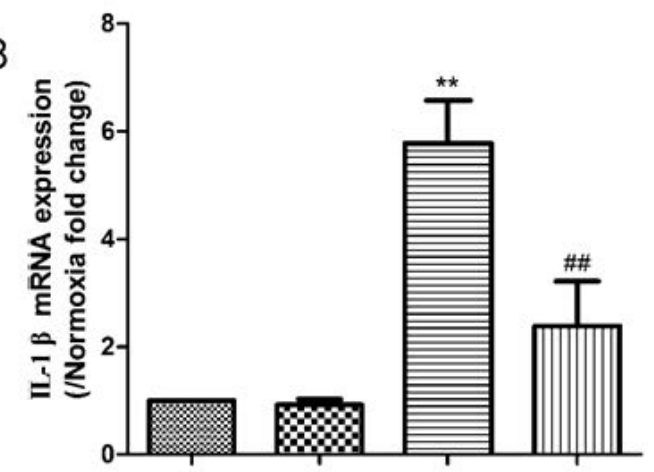

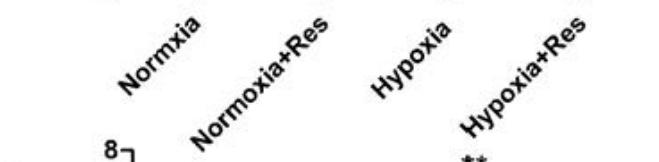

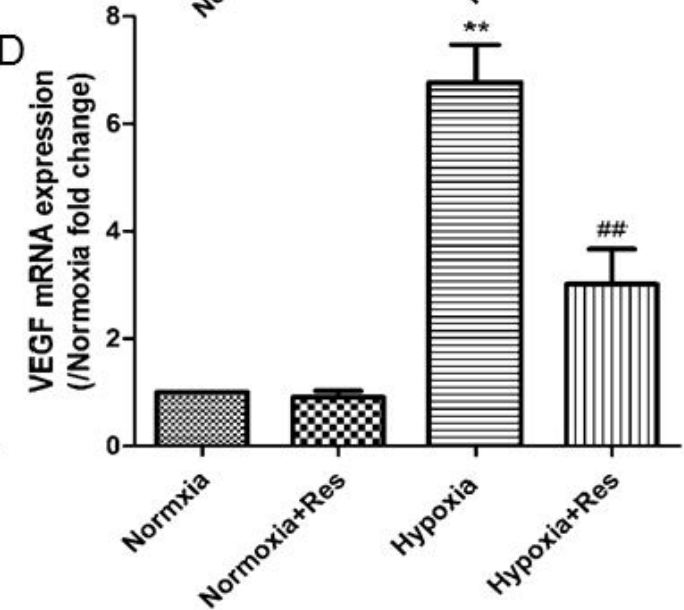

Figure 4. Real time PCR analyses for cytokines of IL-6, IL-1 $1 \beta$, TNF- $\alpha$, and VEGF.

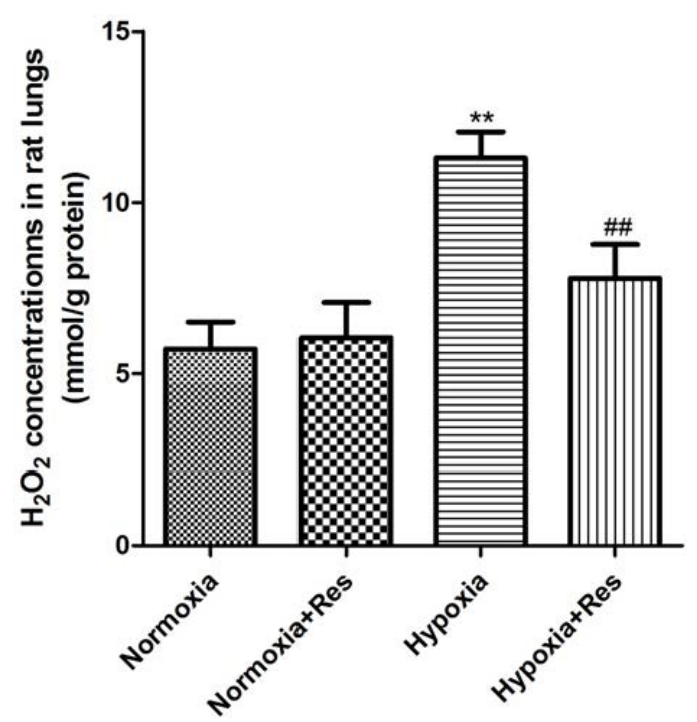

Figure 5. Assays of $\mathrm{H}_{2} \mathrm{O}_{2}$ in rat lungs. 
A

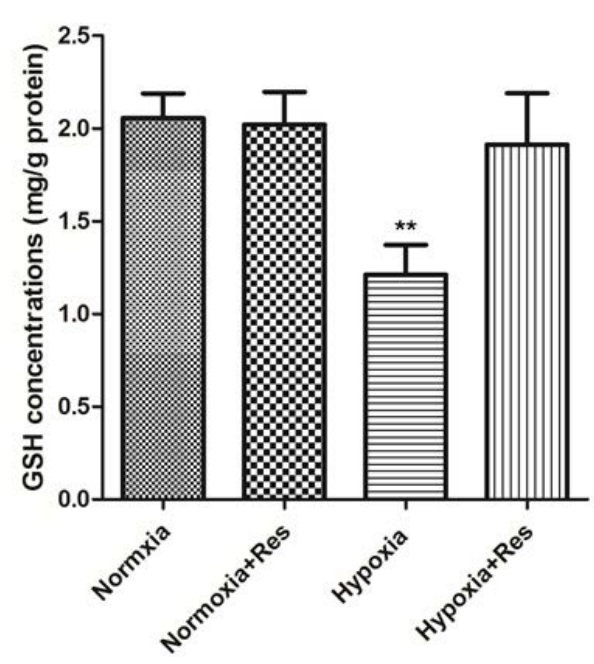

$\mathrm{B}$

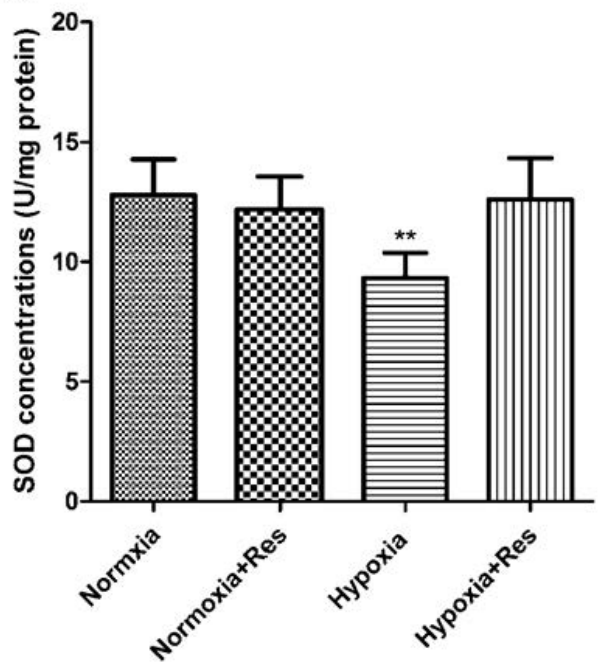

Figure 6. GSH and SOD activities analyses in rat lungs.

A

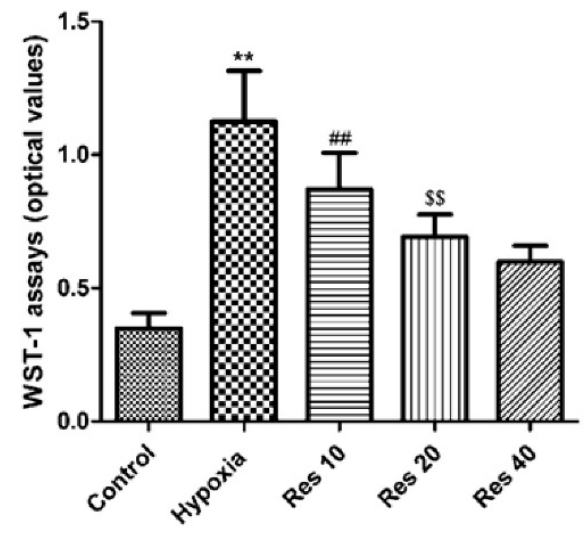

B

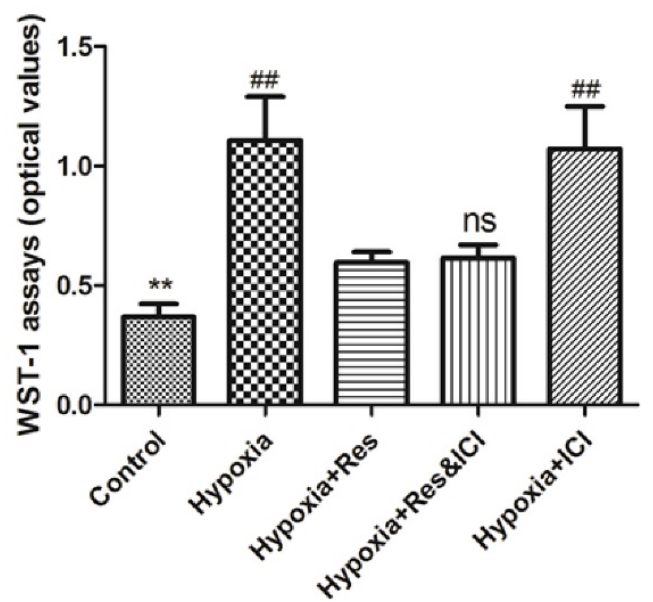

Figure 7. WST-1 assays.

\section{Resveratrol decreased HIF-1 $\alpha$ expression, reversed Trx-1 and Nrf-2 reduction in rat lungs after hypoxia exposure}

As a key factor during hypoxic pulmonary hypertension, HIF-1 a was observed. For further exploring the effects of resveratrol, WB assays were completed. As can see from the data, 28 days hypoxia exposure resulted in great elevation of HIF-1 a in rat lungs versus the normoxic ones $(P<0.01$, Figure 9Aa). Resveratrol treatment significantly decreased the elevation of HIF-1 a expression (compared with hypoxia group, $P<0.01$, Figure 9Aa). However, resveratrol administration did not affect the expression of HIF-1 a notably in the normoxia+res group (VS normoxia group, Figure 9Aa).

However, chronic hypoxia markedly reduced Trx-1 and Nrf-2 protein expression in rat lungs (VS normoxic groups, $P<0.01$, Figure $9 \mathrm{Ab}, \mathrm{Bc}$ ), and resveratrol reversed reduction of both proteins expression $(P<0.01$, VS hypoxia group, Figure $9 \mathrm{Ab}$, $\mathrm{Bc})$. There were no significant differences found in the expression of Trx-1 and Nrf-2 in the two normoxic groups (Figure $9 \mathrm{Ab}, \mathrm{Bc}$ ).

\section{Resveratrol inhibited HIF-1 a expression, increased Trx-1 and Nrf-2 expression in PASMCs}

After hypoxic condition exposure, HIF-1 a protein expression was greatly elevated in the PASMCs compared to the normoxic group $(P<0.01$, Figure 10Aa), and resveratrol treatment dramatically inhibited its expression in a dose-dependent way $(P<0.01$, Figure 10Aa). 

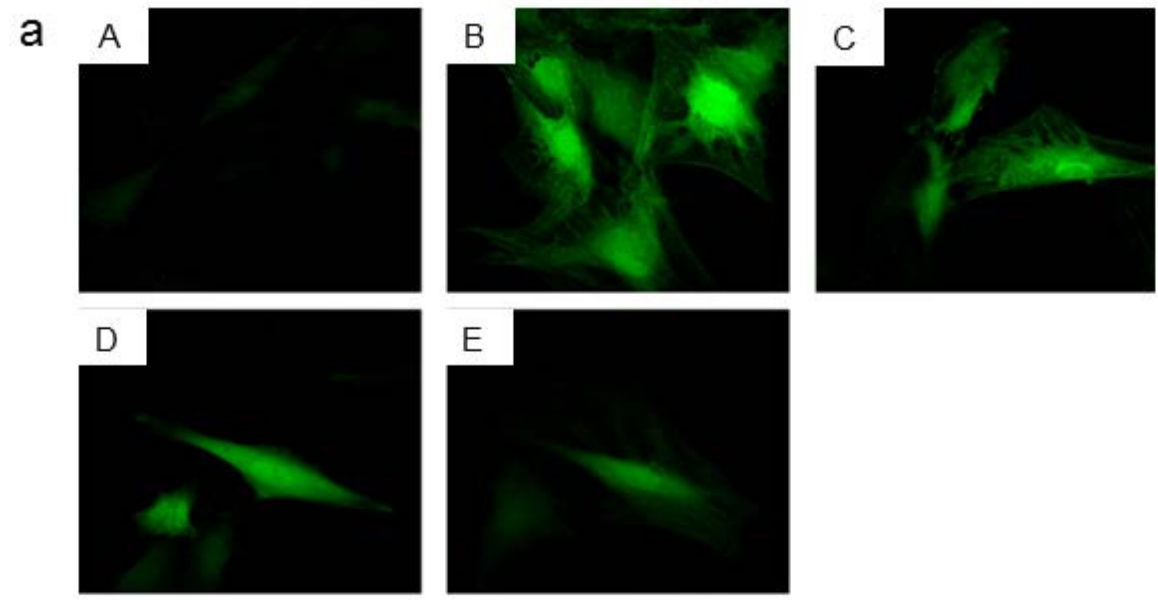

b

C
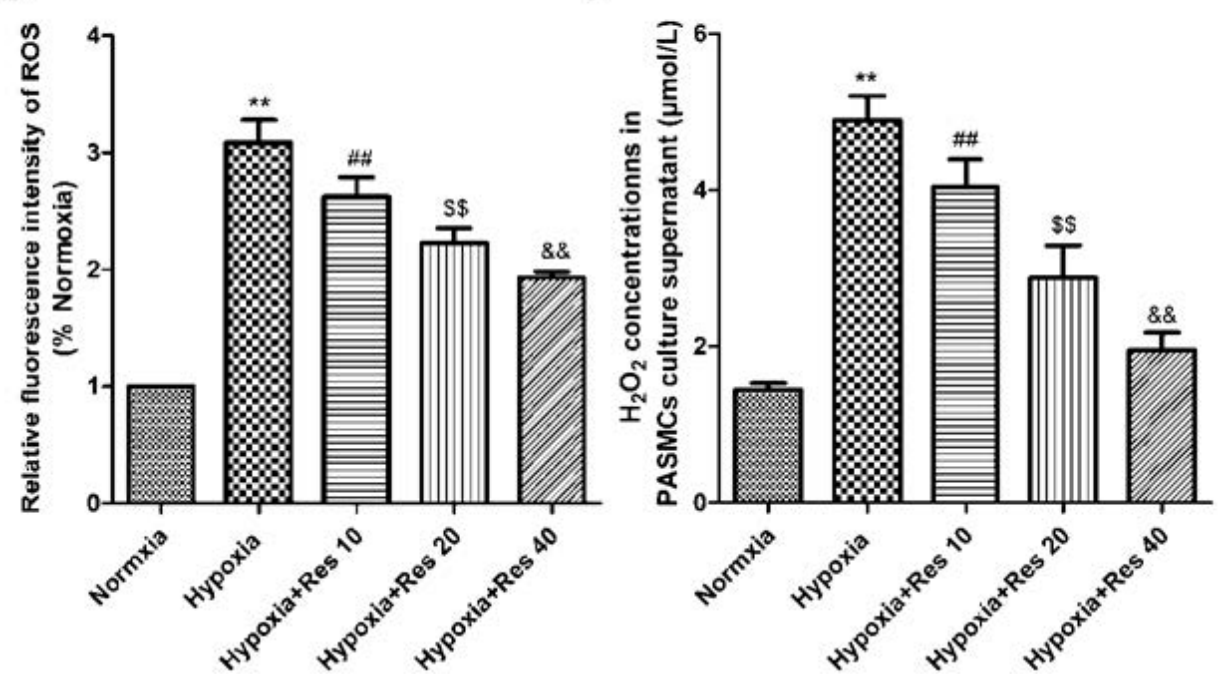

Figure 8. $\mathrm{ROS}$ and $\mathrm{H}_{2} \mathrm{O}_{2}$ detections.
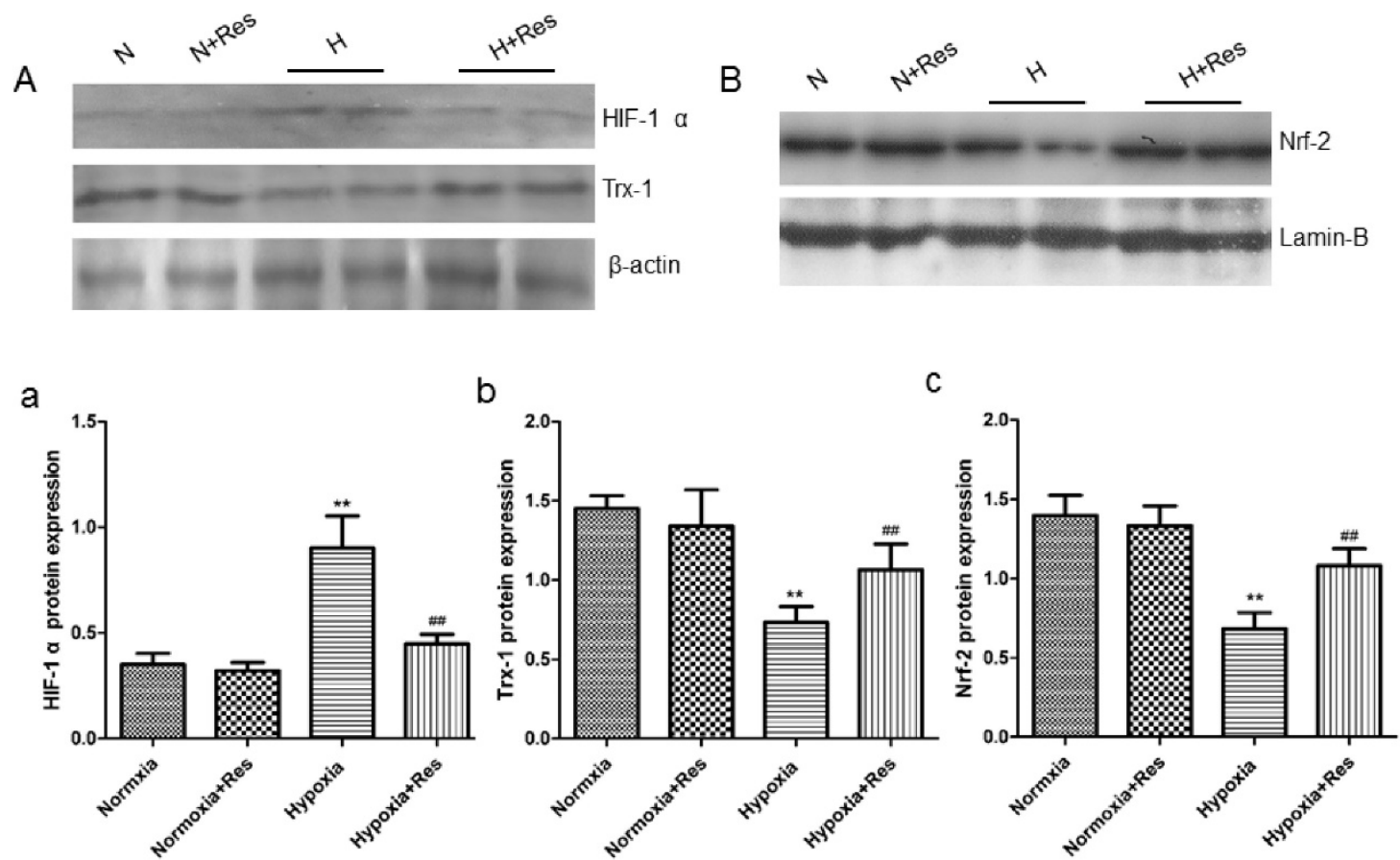

Figure 9. Resveratrol affected the expression of Hif-1 $\alpha$, Trx-1, and Nrf-2 protein levels in rat lungs after chronic hypoxia. 
A
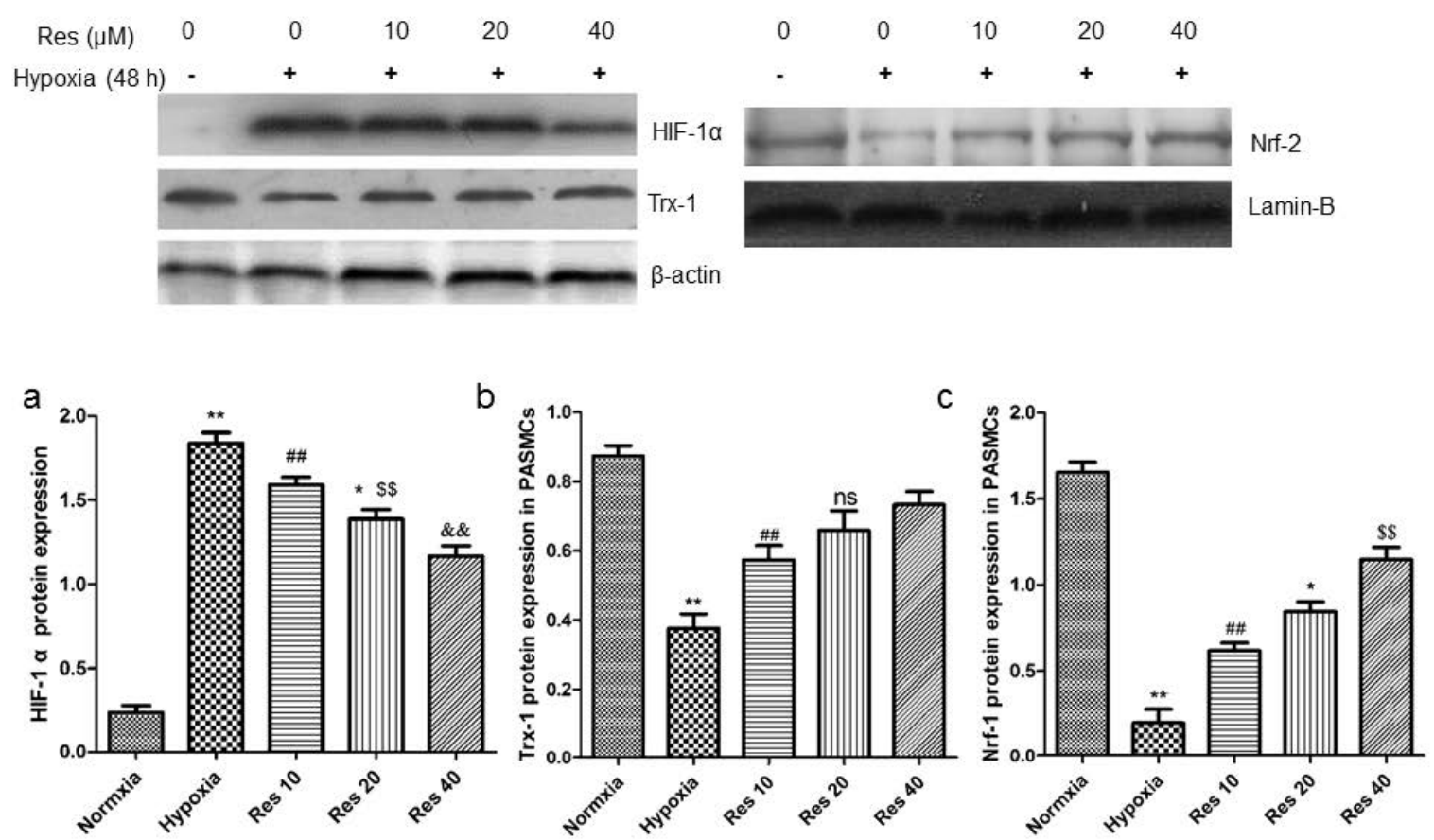

Figure 10. Resveratrol affected the expression of HIF-1 $\alpha, \mathrm{Trx}-1$, and Nrf-2 protein levels in PASMCs after hypoxia exposure.

Moreover, the protein levels of Trx-1 and Nrf-2 were all significantly decreased in PASMCs after hypoxic exposure $(P<0.01$, Figure $10 \mathrm{Ab}, \mathrm{Bc})$. However, resveratrol treatment reversed both of the proteins expression reduction in PASMCs in a dose-dependent way $(P<0.01$, Figure $10 \mathrm{Ab}, \mathrm{Bc})$.

\section{HIF-1 $\alpha$ inhibitor KC7F2 inhibited its expression and PASMCs proliferation induced by hypoxia exposure}

The increased expression of HIF-1a induced by hypoxia exposure was suppressed by administration of HIF-1a inhibitor KC7F2 in a dose-dependent manner (Figure 11A). Along with the suppression of HIF-1a expression, the proliferation of PASMCs resulted by hypoxia was also inhibited by KC7F2 in a dose-dependent way $(P<0.01$, VS hypoxic group, Figure 11B).

\section{PD and LY inhibited HIF-1 $\alpha$ expression, and resveratrol inhibited phosphorylated Akt and erk expression in PASMCs}

Data showed that hypoxia exposure dramatically increased HIF-1a expression in PASMCs compared to the normoxia group $(P<0.01$, Figure 12Aa), and the PI3K/AKT inhibitor and the MAPK/ERK 1 inhibitor, LY294002 and PD98059, both significantly inhibited its expression (VS hypoxia group, $\# \# P<0.01$ and ${ }^{*} P<0.05$, Figure $12 \mathrm{Aa}$ ).
A

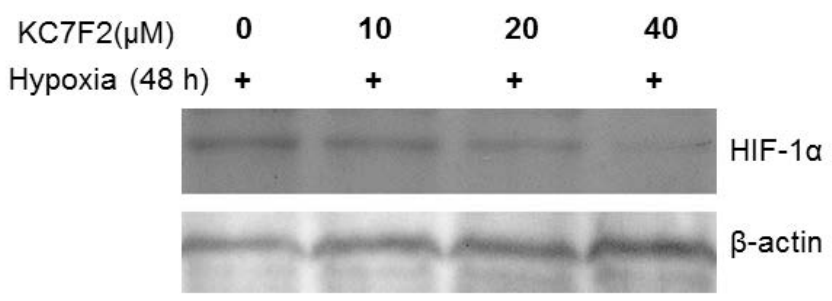

B

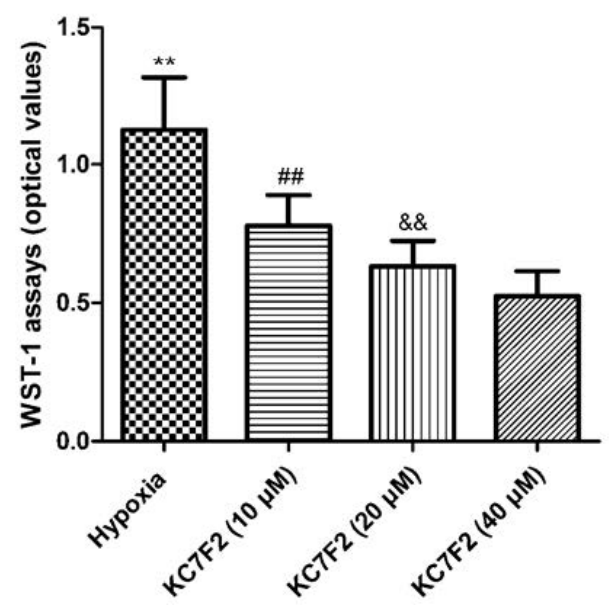

Figure 11. HIF-1 $\alpha$ inhibitor KC7F2 inhibited HIF-1 $\alpha$ expression and PASMCs proliferation. 
A

\section{B}
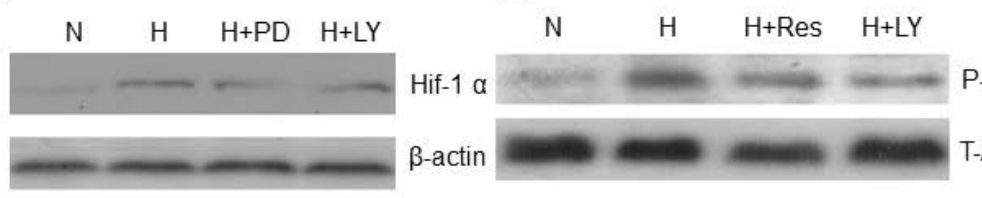

C

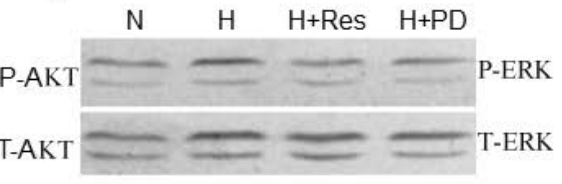

a

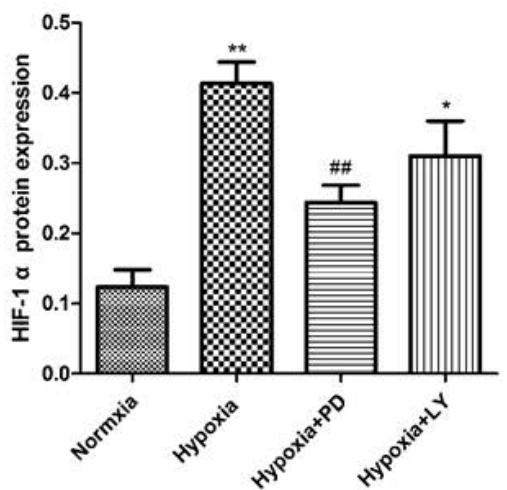

b

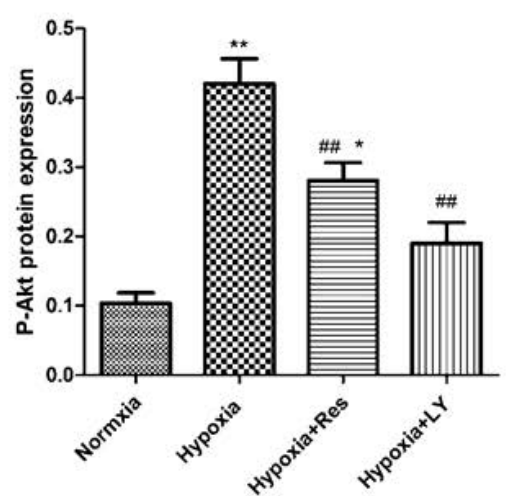

C

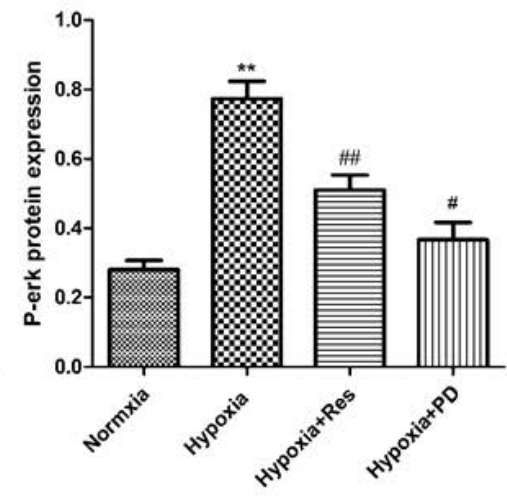

Figure 12. Resveratrol affected the expression of HIF-1 $\alpha$ via inhibiting akt and erk pathways.

Moreover, hypoxia significantly increased the protein level of phosphorylated Akt versus the normoxia group $(P<0.01$, Figure $12 \mathrm{Bb})$, and resveratrol and PD98059 administration both obviously inhibited its elevation (VS hypoxia group, $P<0.01$, Figure 12Bb). Similarly, hypoxia also increased the protein level of phosphorylated erk notably compared to the normoxia group $(P<0.01$, Figure 12Cc), and resveratrol and PD98059 administration both significantly decreased its expression $(P<0.01$, Figure 12Cc).

\section{Discussion}

Resveratrol, a dietary natural phytoestrogen exists in kinds of dark color fruits or red wines, which possesses various pharmacological effects including anti-oxidant, anti-inflammation, modulation of lipid metabolism, and prevention of tumor[1, 2, 13, 23]. In the present study, experimental data displayed that hypoxia-induced significant elevation of right ventricle systolic pressure and right ventricle hypertrophy, deteriorated pulmonary vascular structure remodeling and PASMCs proliferation, which all resemble the characteristics of human pulmonary hypertension. Hence, we successfully established $\mathrm{HPH}$ models of rat. We showed that resveratrol treatment effectively decreased elevation of RVSP, alleviated pulmonary arterioles and right ventricle remodeling in the rat model of chronic hypoxia-induced pulmonary hypertension. The protective effects of resveratrol might include its anti-inflammatory, anti-oxidant and anti-proliferative effects, and the mechanisms underlying these protective effects may ascribe to HIF-1 a and NF-KB signaling pathway inhibition, and Nrf-2/Trx-1 axis regulation.

PASMCs proliferation plays key role in promoting vascular remodeling during the development of $\mathrm{HPH}$, which was significantly suppressed by resveratrol administration. Previous study showed that resveratrol resembles the "non-genomic" protective effects of estrogen via activating ERs in vascular endothelial cells[24]. Other studies also demonstrated that ER-a was involved in the cardiovascular protective effects of resveratrol[25, 26]. However, unlike the studies mentioned above, the present study showed that resveratrol treatment inhibited hypoxia-induced proliferation of PASMCs in an ER-independent manner. In our opinion, the discrepancy may lie in the various biological effects of resveratrol, the different animal disease models, and the different cell types.

Inflammatory response was considered as a new characteristic feature during $\mathrm{HPH}$, and displayed as recruitment and accumulation of inflammatory cells in pulmonary perivascular regions, where remodeling is often prominent[16, 27]. Additionally, animal experiments also showed that oxidative stress plays important roles in the progression of $\mathrm{HPH}[17,28]$. Our previous study (data not published) and other research furthered this viewpoint by showing that administrate possessing anti-oxidative stress and anti-inflammation drugs halt the progression of $\mathrm{PH}[29]$. The present study showed that 
phosphorylated NF-kB expression in rat lungs was inhibited by treating resveratrol, which may suppress its downstream inflammatory cells infiltration. Accordingly, the ED1 staining data of the current study verified that administrating resveratrol suppressed the inflammatory cells infiltration in rat lungs after chronic hypoxia exposure. Additionally, those inflammatory factors genes expression were also greatly inhibited by resveratrol administration. The results of our study added new evidence on the viewpoint that anti-inflammation and anti-oxidative stress method would halt the progression of $\mathrm{PH}$.

Previous studies showed that resveratrol offered protective effects on cardiovascular system via upregulating nuclear factor erythroid-2 related factor 2 (Nrf-2) and thioredoxin-1 (Trx-1)[30, 31]. As a member of transcription factor, Nrf-2 was shown to play a critical coordinator as regulating the redox balance and protecting cells against oxidative and inflammatory lesions[32]. Nrf-2 exerts its balancing effects through regulating the expression of detoxification enzymes and antioxidant proteins, such as HO-1 and Trx-1, in response to a wide range of oxidant/ antioxidant/ electrophilic stimuli to protect the body[33, 34]. Additionally, Nrf-2/Trx-1 axis was demonstrated to modulate apoptosis-related signaling pathway through binding with relevant factors[35]. Our study firstly showed that hypoxia exposure reduced expression of both Nrf- 2 and Trx-1 in vivo and in vitro, and resveratrol treatment significantly reversed the lowered expression of both proteins. On the other hand, our data showed that resveratrol also decreased general ROS production in cultured PASMCs. Similarly, resveratrol reduced $\mathrm{H}_{2} \mathrm{O}_{2}$ production in PASMCs culture supernatant. The decreased ROS production may due to the reversal of Nrf-2/Trx-1 axis by resveratrol administration.

Hypoxia-inducible factors (HIFs) play important roles in response to hypoxia, in which HIF-1 $a$ is the key factor modulating kinds of downstream genes transcription during the progression of $\mathrm{HPH}[36,37]$. The prevailing view of HIF-1 a increase under hypoxia condition is mainly due to its decrease of oxygen-dependent degradation, and increase of oxygen-independent protein synthesis[38]. However, a recent study showed that besides hypoxia, NO and ROS can also activate HIF-1a production[39]. In the current study, ROS and HIF-1 a were both shown to be significantly decreased by resveratrol administration in vivo and in vitro. Moreover, HIF-1 a inhibition by its inhibitor KC7F2 suppressed the proliferation of PASMCs induced by hypoxia. Though the relationship between ROS and HIF-1 a needs to be further explored, the present data intimated that resveratrol prevention of development of $\mathrm{HPH}$ is partially ascribed to decrease of ROS and HIF-1a.

Accumulating evidences have shown that PI3K/AKT pathway activates HIF-1 a in various disease models[40, 41]. Moreover, studies demonstrated that MAPK/ERK1 pathway also involved in activation of HIF-1 a[42-44]. In this study, hypoxia exposure induced HIF-1 a expression in vivo and in vitro, and hypoxia condition also resulted in increase of phosphorylated AKT and ERK expression in cultured PASMCs. In addition, in vitro experiments showed that both ERK1/2 inhibitor PD98059 and the PI3K/AKT inhibitor LY294002 effectively inhibited phosphorylated ERK and AKT expression, together with a significant drop of HIF-1 a expression. Thus, our data confirmed that both MAPK/ERK1 and PI3K/AKT pathways were activated by hypoxia exposure, and then stimulated HIF-1 a activity. Resveratrol treatment significantly inhibited both pathways, through which decreased the expression of HIF-1 a. The suppression of VEGF, a downstream gene of HIF-1 $a$, verified those results. Those data were consistent with the previous study and advanced the mechanisms underlying the protective effects of resveratrol.

Conclusively, our results showed that treatment with resveratrol prevented the development of pulmonary hypertension induced by chronic hypoxia. Those demonstrated anti-proliferation, anti-inflammation, and anti-oxidant actions of resveratrol may answer for its protective effects on HPH. The increased Nrf-2/Trx-1 axis and the decreased HIF-1 a activity through inhibiting MAPK/ERK1 and PI3K/AKT pathways due to resveratrol administration may contribute to the mechanisms beneath those protective effects. These data may suggest potential clinical application of resveratrol on treating $\mathrm{HPH}$.

\section{Abbreviations}

HIF: hypoxia inducible factor; $\mathrm{HPH}$ : hypoxia-induced pulmonary hypertension; NO: nitric oxide; PASMC: pulmonary arterial smooth muscle cell; RVHI: right ventricle hypertrophy index.

\section{Acknowledgement}

Resveratrol is kindly gifted from $\mathrm{Xi}^{\prime}$ an YiLe Bio-Tech laboratory. The authors thank reviewers for English language checking and editing of the paper. This study was supported by the National Natural Science Foundation of China (grants: 81270328, 81471816) and the Project of Natural Science Foundation Research of Shaanxi Province (grant: 2012JM4005). 


\section{Competing Interests}

The authors have declared that no competing interest exists.

\section{References}

1. Shigematsu S, Ishida S, Hara M, et al. Resveratrol, a red wine constituent polyphenol, prevents superoxide-dependent inflammatory responses induced by ischemia/reperfusion, platelet-activating factor, or oxidants. Free Radic Biol Med 2003, 34(7):810-817.

2. Csiszar A, Smith K, Labinskyy N, et al. Resveratrol attenuates TNF-alpha-induced activation of coronary arterial endothelial cells: role of NF-kappaB inhibition. Am J Physiol Heart Circ Physiol 2006, 291(4):H1694-H1699.

3. Clark D, Tuor UI, Thompson R, et al. Protection against recurrent stroke with resveratrol: endothelial protection. PLOS ONE 2012, 7(10):e47792.

4. Carrizzo A, Puca A, Damato A, et al. Resveratrol improves vascular function in patients with hypertension and dyslipidemia by modulating NO metabolism. HYPERTENSION 2013, 62(2):359-366.

5. Stef G, Csiszar A, Lerea K, et al. Resveratrol inhibits aggregation of platelets from high-risk cardiac patients with aspirin resistance. J Cardiovasc Pharmacol 2006, 48(2):1-5.

6. El-Mowafy AM, Alkhalaf M, El-Kashef HA. Resveratrol reverses hydrogen peroxide-induced proliferative effects in human coronary smooth muscle cells: a novel signaling mechanism. ARCH MED RES 2008, 39(2):155-161.

7. Garcia P, Schmiedlin-Ren P, Mathias JS, et al. Resveratrol causes cell cycle arrest, decreased collagen synthesis, and apoptosis in rat intestinal smooth muscle cells. Am J Physiol Gastrointest Liver Physiol 2012, 302(3):G326-G335.

8. Hong SW, Jung KH, Zheng HM, et al. The protective effect of resveratrol on dimethylnitrosamine-induced liver fibrosis in rats. ARCH PHARM RES 2010, 33(4):601-609.

9. Jiang L, Gu Y, Ye J, et al. Resveratrol prevents hepatic steatosis induced by hepatitis C virus core protein. BIOTECHNOL LETT 2012, 34(12):2205-2212.

10. Zhang W, Xue J, Ge M, et al. Resveratrol attenuates hepatotoxicity of rats exposed to arsenic trioxide. FOOD CHEM TOXICOL 2013, 51:87-92.

11. Gupta SC, Kannappan R, Reuter S, et al. Chemosensitization of tumors by resveratrol. Ann N Y Acad Sci 2011, 1215:150-160.

12. Aluyen JK, Ton QN, Tran $\mathrm{T}$, et al. Resveratrol: potential as anticancer agent. J Diet Suppl 2012, 9(1):45-56.

13. Whitlock NC, Baek SJ. The anticancer effects of resveratrol: modulation of transcription factors. NUTR CANCER 2012, 64(4):493-502.

14. Galie N, Hoeper MM, Humbert M, et al. Guidelines for the diagnosis and treatment of pulmonary hypertension. EUR RESPIR J 2009, 34(6):1219-1263.

15. Stenmark KR, Fagan KA, Frid MG. Hypoxia-induced pulmonary vascular remodeling: cellular and molecular mechanisms. CIRC RES 2006, 99(7):675-691.

16. Stenmark KR, Davie NJ, Reeves JT, et al. Frid MG: Hypoxia, leukocytes, and the pulmonary circulation. J Appl Physiol (1985) 2005, 98(2):715-721.

17. Liu JQ, Zelko IN, Erbynn EM, et al. Hypoxic pulmonary hypertension: role of superoxide and NADPH oxidase (gp91phox). Am J Physiol Lung Cell Mol Physiol 2006, 290(1):L2-L10.

18. Xu DQ, Luo Y, Liu Y, et al. Beta-estradiol attenuates hypoxic pulmonary hypertension by stabilizing the expression of p27kip1 in rats. Respir Res 2010, 11:182

19. Lidegaard O, Lokkegaard E, Jensen A, et al. Thrombotic stroke and myocardial infarction with hormonal contraception. N Engl J Med 2012, 366(24):2257-2266.

20. Tofovic SP. Estrogens and development of pulmonary hypertension: interaction of estradiol metabolism and pulmonary vascular disease. J Cardiovasc Pharmacol 2010, 56(6):696-708.

21. Edwards JA, Beck M, Riegger C, et al. Safety of resveratrol with examples for high purity, trans-resveratrol, resVida((R)). Ann N Y Acad Sci 2011, 1215:131-137.

22. Luo Y, Xu DQ, Dong HY, et al. Tanshinone IIA inhibits hypoxia-induced pulmonary artery smooth muscle cell proliferation via Akt/Skp2/p27-associated pathway. PLOS ONE 2013, 8(2):e56774.

23. Ren $\mathrm{Y}, \mathrm{Li} \mathrm{Y}, \mathrm{Zhao} \mathrm{Y}$, et al. Effects of resveratrol on lipid metabolism in C57BL/6J mice. Wei Sheng Yan Jiu 2011, 40(4):495-497.

24. Klinge CM, Blankenship KA, Risinger KE, et al. Resveratrol and estradiol rapidly activate MAPK signaling through estrogen receptors alpha and beta in endothelial cells. J BIOL CHEM 2005, 280(9):7460-7468.

25. Klinge CM, Wickramasinghe NS, Ivanova MM, et al. Resveratrol stimulates nitric oxide production by increasing estrogen receptor alpha-Src-caveolin-1 interaction and phosphorylation in human umbilical vein endothelial cells. FASEB J 2008, 22(7):2185-2197.

26. Khandelwal AR, Hebert VY, Dugas TR. Essential role of ER-alpha-dependent NO production in resveratrol-mediated inhibition of restenosis. Am J Physiol Heart Circ Physiol 2010, 299(5):H1451-H1458.

27. Frid MG, Brunetti JA, Burke DL, et al. Hypoxia-induced pulmonary vascular remodeling requires recruitment of circulating mesenchymal precursors of a monocyte/macrophage lineage. AM J PATHOL 2006, 168(2):659-669.
28. Chovanec M, Novotna J, Wilhelm J, et al. Hypercapnia attenuates hypoxic pulmonary hypertension by inhibiting lung radical injury. PHYSIOL RES 2009, 58 Suppl 2:S79-S85.

29. Csiszar A, Labinskyy N, Olson S, et al. Resveratrol prevents monocrotaline-induced pulmonary hypertension in rats. HYPERTENSION 2009, 54(3):668-675.

30. Ungvari Z, Bagi Z, Feher A, et al. Resveratrol confers endothelial protection via activation of the antioxidant transcription factor Nrf2. Am J Physiol Heart Circ Physiol 2010, 299(1):H18-H24.

31. Kaga S, Zhan L, Matsumoto M, et al. Resveratrol enhances neovascularization in the infarcted rat myocardium through the induction of thioredoxin-1, heme oxygenase- 1 and vascular endothelial growth factor. J MOL CELL CARDIOL 2005, 39(5):813-822.

32. Balligand JL. Reducing damage through Nrf-2. CARDIOVASC RES 2013, 100(1):1-3

33. Go YM, Jones DP. Redox control systems in the nucleus: mechanisms and functions. Antioxid Redox Signal 2010, 13(4):489-509.

34. Harris C, Hansen JM. Nrf2-mediated resistance to oxidant-induced redox disruption in embryos. Birth Defects Res B Dev Reprod Toxicol 2012, 95(3):213-218.

35. Niso-Santano M, Gonzalez-Polo RA, Bravo-San PJ, et al. Activation of apoptosis signal-regulating kinase 1 is a key factor in paraquat-induced cell death: modulation by the Nrf2/Trx axis. Free Radic Biol Med 2010, 48(10):1370-1381.

36. Semenza GL. HIF-1: mediator of physiological and pathophysiological responses to hypoxia. J Appl Physiol (1985) 2000, 88(4):1474-1480.

37. Shimoda LA, Semenza GL. HIF and the lung: role of hypoxia-inducible factors in pulmonary development and disease. Am J Respir Crit Care Med 2011, 183(2):152-156

38. Huang LE, Gu J, Schau M, et al. Regulation of hypoxia-inducible factor 1alpha is mediated by an O2-dependent degradation domain via the ubiquitin-proteasome pathway. Proc Natl Acad Sci U S A 1998, 95(14):7987-7992.

39. Prabhakar NR, Semenza GL. Adaptive and maladaptive cardiorespiratory responses to continuous and intermittent hypoxia mediated by hypoxia-inducible factors 1 and 2. PHYSIOL REV 2012, 92(3):967-1003.

40. Pez F, Dayan F, Durivault J, et al. The HIF-1-inducible lysyl oxidase activates HIF-1 via the Akt pathway in a positive regulation loop and synergizes with HIF-1 in promoting tumor cell growth. CANCER RES 2011, 71(5):1647-1657.

41. Li GQ, Zhang Y, Liu D, et al. PI3 kinase/Akt/HIF-1alpha pathway is associated with hypoxia-induced epithelial-mesenchymal transition in fibroblast-like synoviocytes of rheumatoid arthritis. MOL CELL BIOCHEM 2013, 372(1-2):221-231.

42. Richard DE, Berra E, Gothie E, et al. p42/p44 mitogen-activated protein kinases phosphorylate hypoxia-inducible factor 1alpha (HIF-1alpha) and enhance the transcriptional activity of HIF-1. J BIOL CHEM 1999, 274(46):32631-32637.

43. Du J, Xu R, Hu Z, et al PI3K and ERK-induced Racl activation mediates hypoxia-induced HIF-1alpha expression in MCF-7 breast cancer cells. PLOS ONE 2011, 6(9):e25213.

44. Frede S, Stockmann C, Freitag P, et al. Bacterial lipopolysaccharide induces HIF-1 activation in human monocytes via p44/42 MAPK and NF-kappaB. BIOCHEM J 2006, 396(3):517-527. 